\title{
Modelo de Evaluación basado en TIC para Mejorar el Desempeño del Docente Peruano en el Periodo 2016 - 2018
}

\author{
Evaluation Model based on TIC to Improve the Performance of Peruvian Teachers in 2016 - \\ 2018
}

Pierre Paul Loncán Salazar ${ }^{1}$

\section{RESUMEN}

El objetivo fue proponer un Modelo de Evaluación basado en TIC para Mejorar el Desempeño del Docente Peruano en el Periodo 2016 - 2018. Se realizó una revisión sistemática en las bases de datos: EBSCO Host, ProQuest, Scielo, Google Books, Unesco y repositorios digitales de los Ministerios de Educación de Perú y de otros países. La información se clasificó en concordancia a los aspectos que se deseaban estudiar. Se incluyeron publicaciones de artículos, libros, revistas y diarios. Tras la revisión de todas las publicaciones se encontró diversa información en relación a los modelos de evaluación de desempeño docente, siendo 7 de ellos los más representativos, entre los que se encuentra el modelo de evaluación de desempeño docente de Perú. Entre todos los modelos, se aprecian muchas similitudes, sin embargo cada uno de ellos se enfoca particularmente en algunos aspectos, que a criterio del país al que pertenece el modelo, resulta ser de mayor importancia su evaluación y posterior análisis. Se evidencia un alto grado de importancia para todos los países, el contar con un adecuado sistema o modelo de evaluación de desempeño docente. Para estos modelos es de suma importancia la participación de diversos actores (docentes, alumnos, padres de familia, autoridades educativas y gobierno) para garantizar la adecuada ejecución de estos esquemas de evaluación; sin embargo la realidad muestra que no siempre los resultados obtenidos corresponden plenamente a los resultados esperados.

Palabras clave: Modelo, evaluación, desempeño, docente.

\section{ABSTRACT}

The objective was to propose an Evaluation Model based on TIC to Improve the Performance of the Peruvian Teacher in the 2016-2018 Period. systematic review was done in the databases: EBSCO Host, ProQuest, Scielo, Google Books, Unesco and digital repositories of the Ministries of Education of Peru and other countries. The information was classified according to the aspects that we wanted to study. Publications of articles, books, magazines and newspapers were included. After the review of all the publications, different information was found in relation to the teacher performance evaluation models, 7 of them being the most representative, among which is the teacher performance evaluation model of Peru. Among all the models, many similarities are appreciated, however, each of them focuses in particular on some aspects, which at the discretion of the country to which the model belongs, turns out to be of greater importance its evaluation and subsequent analysis. There is a high degree of importance for all countries, to have an adequate system or model of teacher performance evaluation. For these models, the participation of diverse actors (teachers, students, parents, educational authorities and government) to guarantee the adequate execution of these evaluation schemes; However, reality shows that the results obtained do not always correspond fully to the expected results

Keywords: Model, evaluation, performance, teacher.

\footnotetext{
Universidad Nacional José Faustino Sánchez Carrión. Huacho, Perú.
} 


\section{INTRODUCCIÓN}

La educación es el proceso multidireccional mediante el cual se transmiten: conocimientos, valores, costumbres y formas de actuar (Berger, 2007).

La Educación ha sido y es un elemento importante para la sociedad, ya que permite el desarrollo del ser humano como un individuo con la capacidad de emplear dichos conocimientos, valores, costumbres, etc. en beneficio propio y de la sociedad. Los encargados de llevar a cabo este proceso son los maestros, quienes tienen la misión de brindar una educación que cumpla con los estándares de calidad establecidos (Valdés, 2000).

Con el fin de medir el desempeño de los maestros y constatar que efectivamente se están alcanzado los objetivos educativos planteados, es necesario realizar periódicamente evaluaciones a estos maestros (Reis, 2007). Sin embargo, la realidad demuestra que no siempre la tarea de evaluar es simple de realizar, ya que puede caerse en la subjetividad, alejándose de la objetividad del resultado esperado (Alles, 2004).

Por ello, existen toda clase de metodologías de evaluación de desempeño que pretenden, desde distintos enfoques, tratar de obtener resultados que ayuden a mejorar la educación en sus distintos aspectos (Alles, 2004).

Hoy en día la tecnología ha abarcado muchas áreas, entre las cuales se encuentra la educación. El vínculo formado, tecnologíaeducación ha hecho que ambas evolucionen según los avances tecnológicos y los nuevos usos que se le da a dicha tecnología, en contraste a las actuales necesidades educativas y los nuevos modelos planteados para ellas. El proceso de enseñanzaaprendizaje ya no está restringido a un aula con un grupo de estudiantes y un docente, todos ubicados físicamente en un mismo lugar. Ahora, es posible a través de la tecnología enlazar virtualmente por medio de redes informáticas (Internet, Intranets) a distintos participantes heterogéneos, permitiendo de este modo mejorar su nivel de aprendizaje al compartir conocimientos y experiencias en grupo (Gómez y Lao, 2005).
En el Perú, tal y como nos lo hacen saber Saravia y López de Castilla (2008), la llamada Evaluación de Desempeño Docente se centraba principalmente en evaluaciones de conocimientos sin tomar en cuenta el real desempeño del docente en el aula, es decir, saber si efectivamente los estudiantes están obteniendo los conocimientos esperados. Además, si se considera que en la Ley $\mathrm{N}^{\circ}$ 29062, Congreso de la Republica (2007), se establece que este tipo de evaluaciones deben de realizarse cada 3 años - comparado a otros países en donde las evaluaciones son anuales -, el Estado Peruano a través del Ministerio de Educación (MINEDU, 2012) se ve dificultado en obtener la información oportuna y necesaria para dar una respuesta apropiada ante las verdaderas necesidades de sus docentes.

En la Ley No 29944 del 2012 (MINEDU) actualmente vigente -, sigue existiendo una evaluación basada en conocimientos la cual sigue siendo realizada de forma periódica como máximo cada 3 años, sin embargo, ahora se considera necesaria y esencial la evaluación práctica del docente en el aula frente a los estudiantes.

\section{MATERIAL Y MÉTODOS}

Para realizar la investigación se procedió a la revisión de distintas bases bibliográficas: EBSCO Host, ProQuest, Scielo, Google Books, Unesco y repositorios digitales de los Ministerios de Educación de Perú y de otros países (tanto en inglés como español).

La búsqueda se llevó a cabo usando los siguientes términos: [model OR teacher OR (performance AND evaluation) OR perú] (en inglés) y [modelo OR docente OR (evaluación AND desempeño) OR perú] (en español), incluyéndose en la búsqueda publicaciones de artículos, libros, revistas y diarios.

Se revisaron las publicaciones encontradas que reunían los criterios de búsqueda. Se extrajo información correspondiente al país, año y tipo de publicación, objetivos, criterios e indicadores de evaluación, alcances y limitaciones de evaluación, legislación vigente, fortalezas, deficiencias y evolución de los modelos aplicados. 


\section{RESULTADOS}

Luego de evaluar los documentos recuperados, se obtuvo información acerca de los modelos de evaluación de desempeño aplicados en diversos países, entre ellos el Perú.
Modelo Brasileño: Delannoy (2001) citado por Montenegro (2007) nos menciona que este modelo se enfoca en la carrera profesional del docente basándose en 3 niveles: Básico, Competente y Sobresaliente (Figura 1). Se trata de una serie de certificaciones y promociones que permiten al docente ascender progresivamente de nivel.

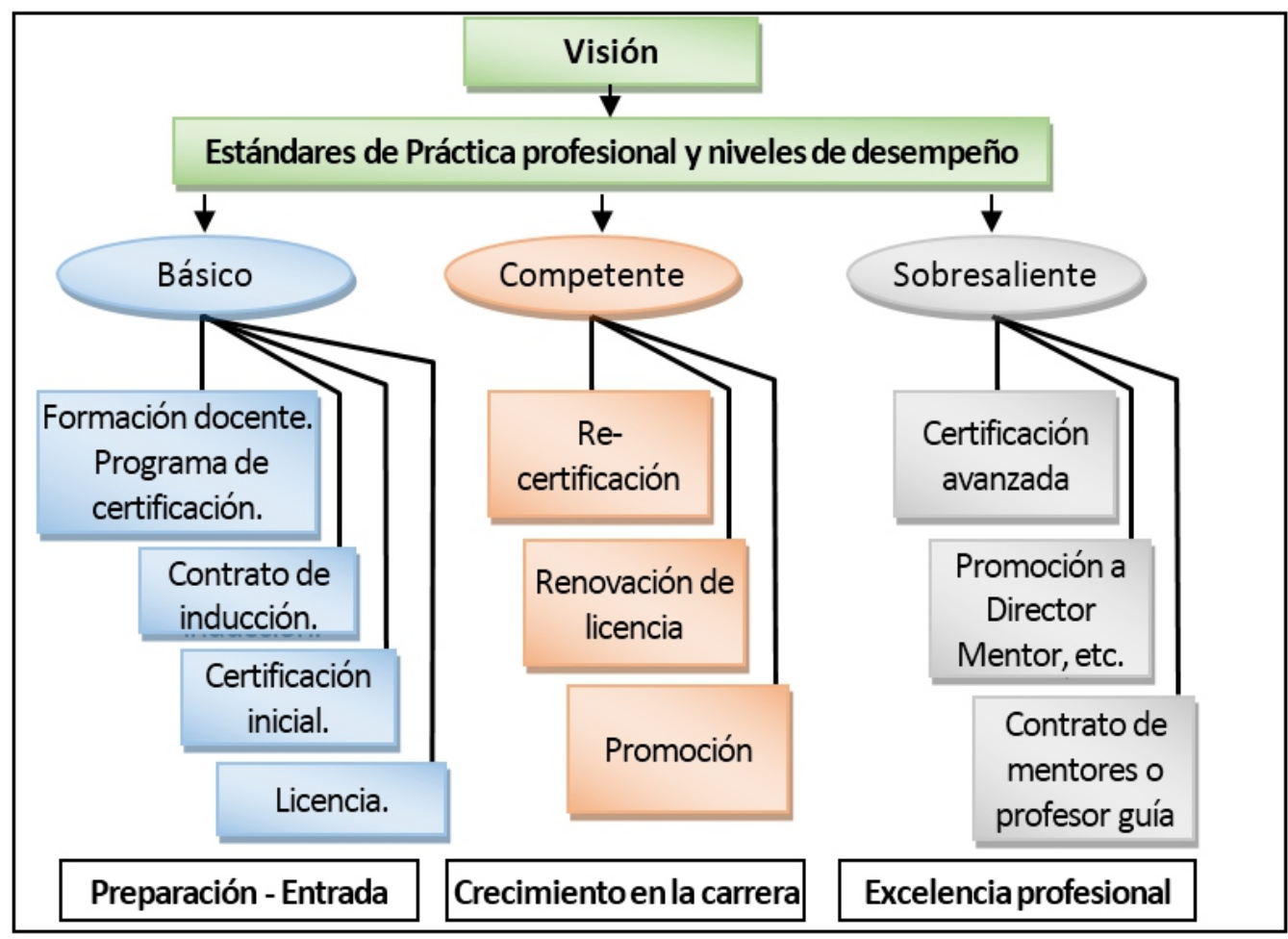

Figura 1. Estructura de la carrera docente organizada en 3 niveles de desempeño Fuente: Montenegro (2007)

Modelo Cubano: Valdés (2000) propone un Modelo de Evaluación de Desempeño Profesional Docente el cual consta de 5
Dimensiones. La muestra la representación de este modelo articulando las dimensiones.

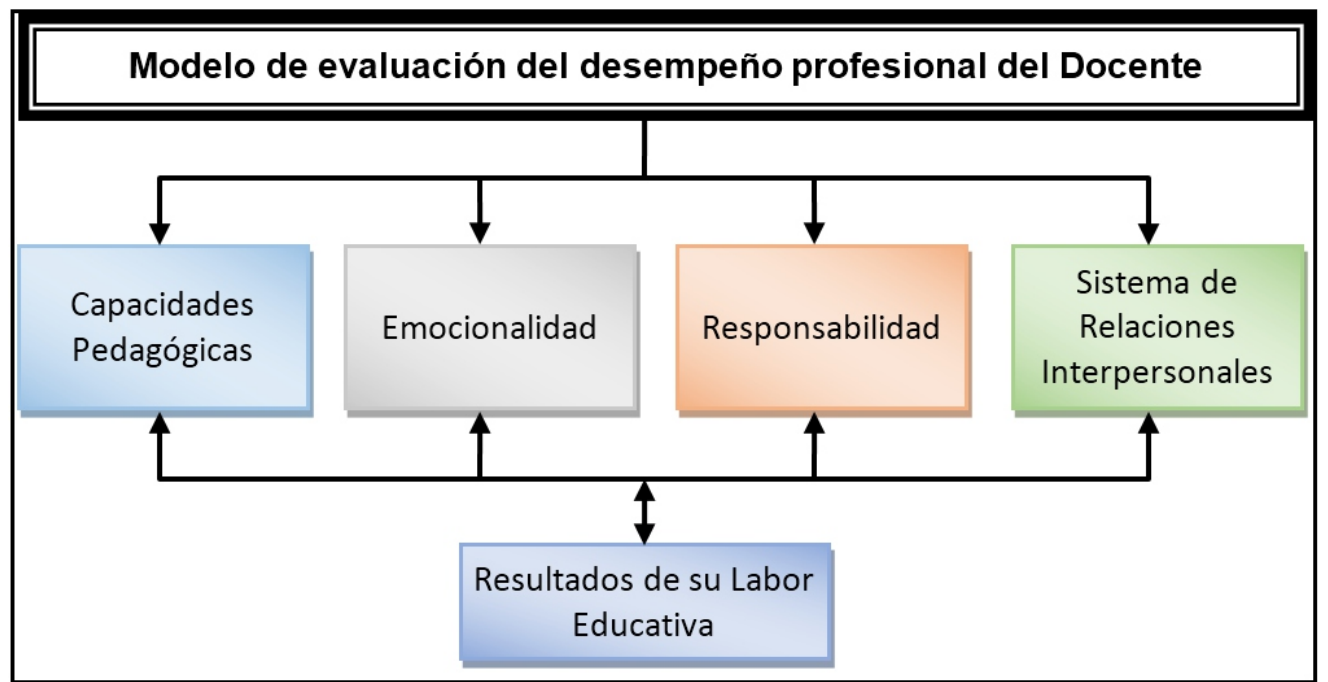

Figura 2. Modelo de Evaluación de Desempeño Profesional Docente Fuente: Valdés (2000) 
Modelo Chileno: Murillo, González y Rizo (2007) la OREALC/UNESCO (2007) habla del Nuevo Sistema de Evaluación de Desempeño Docente el cual fue puesto en marcha en agosto del 2003. Esta evaluación se realiza cada cuatro años, y tiene por finalidad contar con una apreciación global relativa al desempeño profesional del docente, relacionado con el Marco para la Buena
Enseñanza. La Figura 3 muestra los 4 dominios que son considerados como parte de la evaluación del docente. La evaluación se realiza a través de los siguientes instrumentos: portafolio docente, autoevaluación, entrevista estructurada e informe de referencia de terceros. Este Sistema de Evaluación tiene como base la concesión de incrementos salariales

\section{Preparación de la Enseñanza}

A1. Domina los contenidos de las disciplinas que enseña y el marco curricular nacional.

A2. Conoce las características, conocimientos y experiencias de sus estudiantes.

A3. Domina la didáctica de las disciplinas que enseña.

A4. Organiza los objetivos y contenidos de manera coherente con el marco curricular y las particularidades de sus alumnos.

A5. Las estrategias de evaluación son coherentes con los objetivos de aprendizaje, la disciplina que enseña, el marco curricular nacional y permite a todos los alumnos demostrar lo aprendido.

Creación de un ambiente propicio para el aprendizaje

B1. Establece un clima de relaciones de aceptación, equidad, confianza, solidaridad y respeto.

B2. Manifiesta altas expectativas sobre las posibilidades de aprendizaje y desarrollo de todos sus alumnos.

B3. Establece y mantiene normas consistentes de convivencia en el aula.

B4. Establece un ambiente organizado de trabajo y dispone los espacios y recursos en función de los aprendizajes.

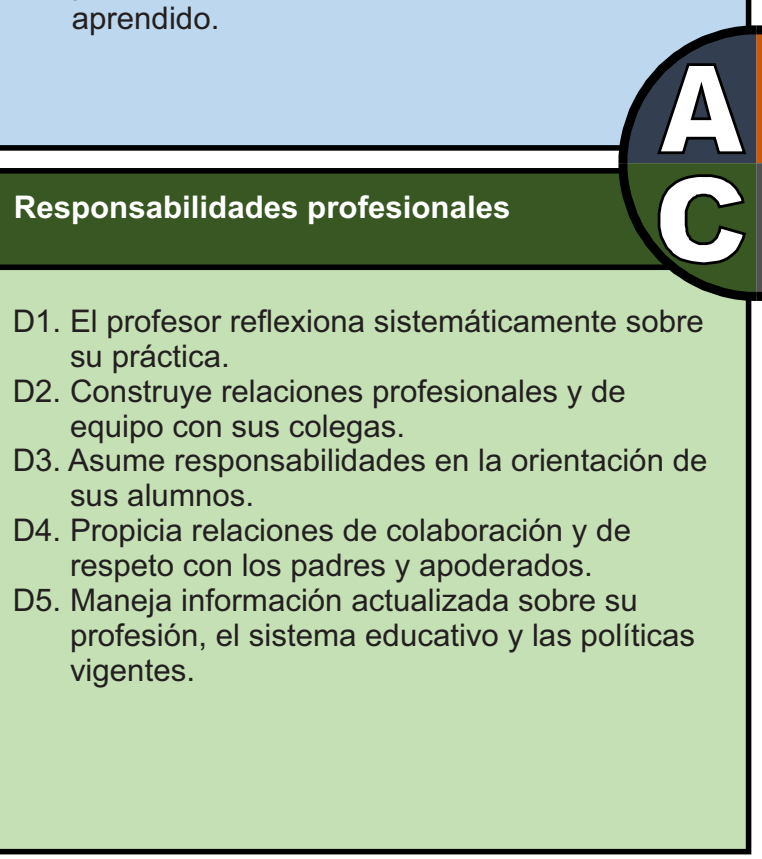

Figura 3. Marco para la Buena Enseñanza del Ministerio de Educación de Chile Fuente: Murillo, González y Rizo (2007) la OREALC/UNESCO

Modelo del Reino Unido: Montenegro (2007) menciona que para el Reino Unido la evaluación de desempeño docente ha surgido a raíz de evaluaciones periódicas que se realizan a los estudiantes. El sistema de evaluación, al igual que en otros países se encuentra en proceso de elaboración y está muy ligado al desarrollo profesional. Entre algunos de los principales elementos del sistema tenemos los siguientes:
Enseñanza para el aprendizaje de todos los estudiantes

C1. Comunica en forma clara y precisa los objetivos de aprendizaje.

C2. Las estrategias de enseñanza son desafiantes, coherentes y significativas para los estudiantes.

C3. El contenido de la clase es tratado con rigurosidad conceptual y es comprensible para los estudiantes.

C4. Optimiza el tiempo disponible para la enseñanza.

C5.Promueve el desarrollo del pensamiento.

C6. Evalúa y monitorea el proceso de comprensión y apropiación de los contenidos por parte de los estudiantes.

- Evaluación rigurosa durante la formación inicial y final de ella, la cual se compone de una evaluación práctica y prueba escrita.

- Determinación de un perfil de entrada a cada nuevo profesor calificado.

- Evaluación al año de la inducción dirigida por la escuela.

- Desarrollo de un marco nacional de 
calificaciones profesionales.

- Incentivos económicos especiales para los profesores excepcionales.

- Desarrollo profesional de los profesores liderado por profesores avanzados.

- Implementación de nuevos tipos de desarrollo profesional.

- Elaboración de estándares profesionales.

- Etc.
Modelo de Estados Unidos: Montenegro (2007) explica que en este modelo se aplica una Prueba de Certificación de PRAXIS. La Tabla 1 muestra los 4 aspectos que forman parte de este modelo y para cada uno de estos aspectos existe una relación de componentes o indicadores que se tomarán en cuenta durante el proceso de evaluación.

Tabla 1. Estructura de los contenidos de la prueba de Certificación de PRAXIS

\begin{tabular}{|c|c|c|}
\hline & Aspectos & Componentes \\
\hline 1. & Planificación y preparación & $\begin{array}{l}\text { Demostrar conocimiento de contenidos y pedagogía } \\
\text { Demostrar conocimiento de los alumnos } \\
\text { Seleccionar objetos instruccionales } \\
\text { Demostrar conocimiento de los recursos } \\
\text { Evaluar el aprendizaje del alumno }\end{array}$ \\
\hline 2. & El ambiente en la sala de clases & $\begin{array}{l}\text { Crear un ambiente de respeto y armonía } \\
\text { Establecer una cultura para el aprendizaje } \\
\text { Administrar los procedimientos de aula } \\
\text { Manejar la conducta del alumno } \\
\text { Organizar el espacio físico }\end{array}$ \\
\hline 3. & Instrucción & $\begin{array}{l}\text { Comunicar en forma clara y precisa } \\
\text { Usa técnicas de interrogación y discusión } \\
\text { Comprometer a los alumnos en el aprendizaje } \\
\text { Entregar retroalimentación a los alumnos } \\
\text { Demostrara flexibilidad y sensibilidad }\end{array}$ \\
\hline 4. & Responsabilidades Profesionales & $\begin{array}{l}\text { Reflexionar sobre la enseñanza } \\
\text { Mantener registros precisos } \\
\text { Comunicarse con las familias } \\
\text { Contribuir con la escuela y el distrito } \\
\text { Crecer y desarrollarse profesionalmente } \\
\text { Demostrar profesionalismo }\end{array}$ \\
\hline
\end{tabular}

Fuente: Montenegro (2007)

Modelo colombiano: Montenegro (2007) y Murillo, González y Rizo (2007) la Oficina Regional de Educación de la OREALC/UNESCO nos hablan del modelo colombiano el cual a partir del 2003 entró en vigencia. La evaluación verifica el desempeño de docentes y directivos determinando así su permanencia en el cargo y ascensos en el escalafón, así como también las reubicaciones en los niveles salariales. La Tabla 2 muestra la normativa que establece 14 aspectos del desempeño a valorar, cada uno de los cuales es calificado dentro de la siguiente escala: Inferior (1 o 2 puntos), Medio (3 o 4 puntos) y Superior (5 o 6 puntos).

Tabla 2. Aspectos a valora del Desempeño Docente

Aspectos a valorar del Desempeño Docente
Construcción y Desarrollo del Proyecto Educativo Institucional (PEI)
Cumplimiento de las normas y políticas educativas
Conocimiento y valoración de los estudiantes
Fundamentación pedagógica
Planificación del trabajo
Estrategias pedagógicas
Estrategias para la participación
Evaluación y mejoramiento
Innovación
Compromiso institucional
Relaciones interpersonales
Mediación de conflictos
Trabajo en equipo
Liderazgo

Fuente: Murillo, González y Rizo (2007), la OREALC/UNESCO 
Modelo Peruano: En el caso de Perú (MINEDU 2012), no se habla explícitamente de un Modelo de Evaluación de Desempeño, en su lugar se tiene el "Marco del Buen Desempeño Docente" basado en 4 dominios, que a partir del año 2012 junto con la promulgación de la Ley de Reforma Magisterial establecen los lineamientos para realizar la evaluación de los docentes. Los cuatro dominios aquí mencionados comprenden a su vez nueve competencias (Ver Tabla 3) que a su vez contemplan 40 desempeños.

\section{Tabla 3. Competencias correspondientes al Marco del Buen Desempeño Docente}

\section{Dominlo I: Preparacion para el a prendzaje de lo5 es tudlantes}

\section{Competenclas}

1. Conoce y comprende las caracteristicas de tod $0 s$ sus estudlantes y sus contextos, los contenidos disclpilnares que enseta, los enfoques y proces $0 s$ pedagogloos, con el propos tho de promover capacidades de anto nivel y su formacion integral.

2. Planinca la ensefianza de forma colegada garantizando la conerencla entre los aprendlajes que qulere lograr en sus estudantes, el proceso pedagoglos, el uso de los recursos dlsponibles y la evaluacion, en una programacion curficular en perm anente revis ion

\section{Dominlo II: Ense nanza para el a prendizaje de los es tudla ntes}

\section{Competenclas}

3. Crea un cllm a propiclo para el aprendlaje, la convivencla dem ocratica y la vivencla de la diversidad en todas sus expreslones, con miras a form arcludadanos crticos e intercu hurales.

4. Conduce el proceso de ensedianze con dominlo de los contenldos disclpllnares y el uso de estrategas y recursos pertinentes para que todos los estudlantes aprendan de manera reflexiva y crtica todo lo que conclene a la soluclon de problem as relaclonados con $8 u s$ experlenclas, intereses y contextos cu ifurales.

5. Evalda permanentemente el aprendlzaje de acuerdo con los objetwos insthuclonales prevlstos, para tomar declsiones y retroallmentar a sus estudlantes y a la comunidad educativa, tenlendo en cuenta las diferenclas Ind Mouales y los dlversos contextos cururales.

\section{Dominio a: Participacion en la gestion de la escuela articulada a la comunidad}

\section{Competenclas}

6. Particlpa activam ente con actiud dem ocratica, crtica y colaborativa en la gestion de la escuela, contribuyendo a la construccilon y mejora continua del Proyecto Educativo insthuclonal para que genere aprendlajes de callibd.

7. Establece relaciones de respeto, colaboracion y comesponsabllidad con las fam llias, la comunload y otias Insthuclones del Estado y la socledad clvil.

8. Aprovecha 8 s saberes y recurs 06 en los procesos educativos y da cuenta de los resultados.

\section{Dominio N: Desarrollo de la protesilonalidady la ldentidad docente}

\section{Competencia 5}

9. Reflexiona sobre su practica y experlencla insthuclonal y desarrolla procesos de aprend lzaje continuo de m odo Ind Midual y colectivo, para construlir y afirm ar 8 u identidad y responsabilldad profesional.

10. Ejerce su profesion desdeuna etica de respebo de los derechos fundamentales de las personas, demostrando honestidad, Justicla, responsabllidad y compromlso con su funcion soclal.

Fuente: MINEDU (2012a) 


\section{CONCLUSIONES}

Tras la revisión bibliográfica se ha apreciado que en todos los países existe una constante preocupación por medir y hacer el seguimiento del desempeño del docente. Por ello, han elaborado e implementado modelos de evaluación que buscan satisfacer esta necesidad.

Los distintos modelos encontrados comparten muchas características, entre las que podemos mencionar: (1) la planificación y preparación del aprendizaje para que cada docente prepare y adecue la enseñanza a partir de las necesidades reales de sus estudiantes tomando como base el contexto social, económico, cultural y académico de cada uno de ellos , (2) el clima o ambiente en el aula para garantizar un adecuado desarrollo de las actividades académicas a través de un constante seguimiento del progreso de sus estudiantes tanto desde el aspecto académico como también desde el social con lo cual el docente ajusta de forma permanente los recursos y estrategias utilizadas como parte de su labor diaria, (3) la participación en la gestión institucional que incentive al docente a colaborar activamente en la mejora continua del proyecto educativo institucional y (4) el desarrollo profesional que garantice la mejora continua del docente tanto en el ámbito profesional como en las relaciones interpersonales.

Es apreciable que Perú cuenta, a través del llamado "Marco del Buen Desempeño Docente", con las 4 características descritas en el párrafo anterior, lo cual nos daría a entender que existe una propuesta razonable a partir sobre la cual es posible diseñar un "Modelo de Evaluación basado en TIC para Mejorar el Desempeño del Docente Peruano".

\section{REFERENCIAS BIBLIOGRÁFICAS}

Alles, M. (2004). Diccionario de comportamientos: gestión por competencias: cómo descubrir las competencias a través de los comportamientos. Ediciones Granica SA.
Berger, K. S. (2007). Psicología del desarrollo: infancia y adolescencia ( $7^{\circ}$ ed.). Ed. Médica Panamericana.

Congreso de la Republica (2007). Ley 29062. Ley que modifica la Ley del Profesorado en lo referido a la Carrera Publica Magisterial. Diario Oficial El Peruano, $348857-348866$.

Gómez, M. E. P., \& Lao, J. M. I. (2005). La dinámica del proceso docente educativo en la educación superior, con el empleo de las tecnologías de la información y las comunicaciones. Pedagogía Universitaria, 10(5), 83-96.

MINEDU (2012, noviembre, 25). Ley 29944 Ley de Reforma Magisterial. Diario Oficial El Peruano, 479340-479350.

MINEDU. (2012a, Diciembre, 27). Se aprobó la Resolución Ministerial N 0547- 2012ED. Marco de Buen Desempeño Docente para Docentes de Educación Básica Regular". Documento de trabajo. Lima, Perú. Recuperado, de http://www.minedu.gob.pe/n/xtras/marc o_buen_desempeno_docente.pdf

MINEDU (2013, Mayo, 03). Aprueban Reglamento de la Ley N²9944, Ley de Reforma de Reforma Magisterial $\mathrm{N}^{\circ}$ 29944. Reglamento de la Ley de Reforma Magisterial, D.S.N 004-2013ED. Diario Oficial El Peruano, $494061-$ 494084.

Montenegro, I.A. (2007). Evaluación del Desempeño Docente. Fundamentos, Modelos e Instrumentos. (2da Ed.). Bogotá: Cooperativa Editorial Magisterio.

Murillo Torrecilla, J. F., González de Alba, V., \& Rizo Moreno, H. (2007). Evaluación del Desempeño y Carrera Profesional Docente: Un estudio comparado entre 50 países de América Latina y Europa. Oficina Regional de Educación de la UNESCO para América Latina y el Caribe OREALC/UNESCO Santiago. Recuperado de http://unesdoc. unesco. org/images/0015/001529/152934s. pdf. 
Reis, P. (2007). Evaluación de Desempeño. Madrid: VerlagDashöfer Ediciones Profesionales, S. L. U.

Saravia L. \& López de Castilla, M. (2008). La Evaluación del Desempeño Docente. Perú, una Experiencia en Construcción. RIEE. Revista Iberoamericana de Evaluación Educativa.

Valdés Veloz, H (2000). En un mundo de cambios rápidos, sólo el fomento de la innovación en las escuelas permitirá al sistema educacional mantenerse al día con los otros sectores. Ponencia presentada en el Encuentro Iberoamericano sobre Evaluación del Desempeño Docente. OEl, México, del, 23 al 25 de mayo.
Correo electrónico:

ploncan24@hotmail.com

Revisión de pares:

Recibido:

Aceptado: 